\title{
Literature contributions of a paediatric cardiologist to adult congenital heart disease - Part II
}

\author{
P. Syamasundar Rao \\ Professor of Pediatrics \& Medicine, Emeritus Chief of Pediatric Cardiology, University of Texas at Houston, McGovern Medical School, USA
}

\begin{abstract}
The author's contributions with regard to defects in the atrial septum, namely, secundum atrial septal defects (ASDs) and patent foramen ovlae (PFO) will be reviewed. Successful closure with the buttoned device with excellent immediate and follow-up results was demonstrated in patients with 1. ostium secundum ASDs, 2. patent foramen ovale (PFO) presumed to be the seat of paradoxical embolism, 3. residual ASDs/PFOs with right-to-left shunt following prior cardiac surgery, and 4. ASDs/ PFOs resulting in platypnea-orthodeoxia syndrome. Also reviewed was why, when and how should ASDs be closed in adults. Since the buttoned device is no longer available for clinical use, now the author utilizes other devices, namely, Amplatzer and Gore Septal occluders instead.
\end{abstract}

\section{Introduction}

In the introduction section of Part I of these reviews, the author's contributions to the literature in relation to adult congenital heart defects (CHDs) were enumerated and the increasing prevalence of CHDs in adult population was pointed out. Part I reviewed aortopulmonary communications, namely patent ductus arteriosus and aortopulmonary windows. In this Part II, defects in the atrial septum, namely, secundum atrial septal defects (ASDs) and patent foramen ovlae (PFO) will be reviewed.

\section{Transcatheter occlusion of atrial septal defect}

The results of transcatheter occlusion of secundum ASDs in adults was combined with those of the results in children [1-8]. A total of 768 patients had buttoned device implantation to occlude ostium secundum ASDs from 1989 to 2002 at 40 institutions around the world $[1,5,6,9,10]$. These patients were divided in three cohorts on the basis of the type of buttoned device implanted. Cohort I consisted of 180 patients in whom single-button (first, second \& third generation) (Figures 1 and $2 \mathrm{~A} \&$ B) buttoned device implantations were performed between 1989 and 1993. Cohort II is made up of 423 patients in whom double-button (fourth generation) (Figures $2 \mathrm{C}$ and 3 ) buttoned device placements were performed between 1993 and 1997. Cohort III is composed of 175 patients in whom centering-on-demand (fourth generation with centering mechanism) (Figure 4) buttoned device implantations were performed from 1999 to 2002. The percentage of adult subjects was $18 \%$ in Cohort I, $43 \%$ in Cohort II, and 29\% in Cohort III. In general, the results in adults were similar to those seen for the entire groups $[1,5,6,8]$. A careful comparison of adult subjects in Cohorts I and II [8] revealed a decrease in unbuttoning rates $(12 \%$ versus $1.6 \%$; $<0.01)$ while equal effectiveness was maintained in the occlusion rates ( $96 \%$ vs. $85 \% ; \mathrm{p}>0.1$ ). The re-intervention rates during follow-up also appeared to decrease $(11 \%$ vs. $3.5 \%$; $<$ < 0.05$)$. In the adult subjects of Cohort III, there was continued improvement in feasibility and effectiveness. The results of buttoned-device occlusion in adult subjects were similar to those seen for the entire group, and for children, with progressive improvement in device performance occurring with successive modifications of the buttoned device [8].

\section{Why should ASDs be closed in adults}

In the past, several authorities [11-13] have suggested that the closure of ASDs in adult subjects is not necessary unless they are symptomatic. The author's opinion is contrary to such recommendations and has reviewed the available data to see if they support of closure of ASDs in adults even if they are not symptomatic $[14,15]$. These reviews indicated that 1. untreated adult ASD patients tend to have decreased eventfree survival rates when compared to the normal population [16],2. surgical closure is safe and effective, with high event-free survival rates [17,18], 3 . ASD closure prevents functional deterioration [19], 4. The closure of an ASD improves cardiac function [20], and 5. ASD occlusion increases functional capacity [21]. Consequently, it was concluded that hemodynamically significant ASDs in all adult patients should undergo closure [14,15].

\section{When should ASDs be closed in adults?}

Murphy and colleagues [22] studied the effect of age at the time of surgical closure of ASD. The outcome of 123 patients who had surgical closure of ASD (procedure performed between 1956 and 1960) at Mayo Clinic were examined. The actuarial survival rates of these 123 ASD patients were compared with those of normal populations of comparable ages. The actuarial survival rates were lower (Figure 5) in patients who had surgery after 24 years of age than those of normal population. However, when surgery was undertaken before the age of 24 years, there was no significant difference in survival rates. The earlier the surgery was performed the better was the 27-year survival rate (Figure 5). Murphy et al concluded that early intervention may be

*Correspondence to: P Syamasundar Rao, Professor of Pediatrics \& Medicine, Emeritus Chief of Pediatric Cardiology, University of Texas at Houston, McGovern Medical School, Houston, Texas, USA, Tel: 832-2691050, E-mail: P.Syamasundar.Rao@uth.tmc.edu

Key words: atrial septal defect, patent foramen ovlae, percutaneous occlusion, buttoned device, residual shunts, paradoxical embolism, Amplatzer septal occluders, septal occluders

Received: July 07, 2020; Accepted: July 17, 2020; Published: July 22, 2020 
beneficial. Therefore, we recommend that closure of hemodynamically significant ASDs in all adults be undertaken at the time of their presentation $[14,15]$.

\section{How should ASDs be closed in adults?}

Following the first description of surgical closure of ASD by Kirklin and his associates in the mid-1950s [23], it rapidly became a standard

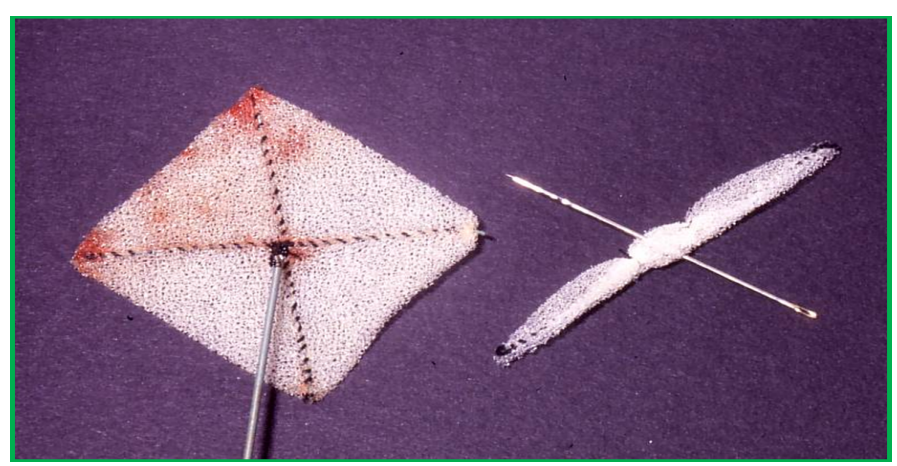

Figure 1. Photograph of the original buttoned device

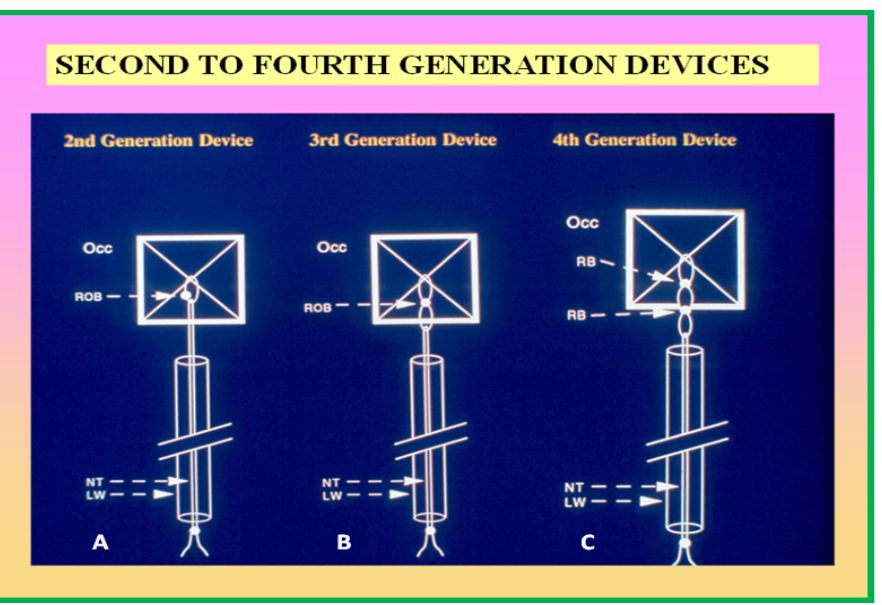

Figure 2. Cartoon depicting the occluder component of the second-, third- and fourthgeneration buttoned devices. The occluder (Occ) in all devices is composed of an x-shaped wire skeleton covered with polyurethane foam. In the first-generation device (Figure 1), the button was not radiopaque. In the second-generation device (left) a $2 \mathrm{~mm}$ string loop is attached to the center of the occluder. The loop is closed with a knot (button) and made radiopaque. This radiopaque button (ROB) can easily be visualized by fluoroscopy. A folded 0.008 " nylon thread (NT) passes through the hollow loading wire (LW) after passing through the loop in the center of the occluder. In the third-generation device (middle) an extra loop is added immediately beneath the radiopaque button, making the eccentric button of the second-generation device to be aligned straight, thus making it easier to button the Occ and counter-occluder across the atrial septum. In the fourth-generation device (right), the button loop is replaced with two "spring" radiopaque buttons (RB), mounted $4 \mathrm{~mm}$ apart. The intent of these modifications was to reduce the unbuttoning seen with earlier generation devices. Modified from Rao PS, et al., J Am Coll Cardiol 2000; 36:583-92.

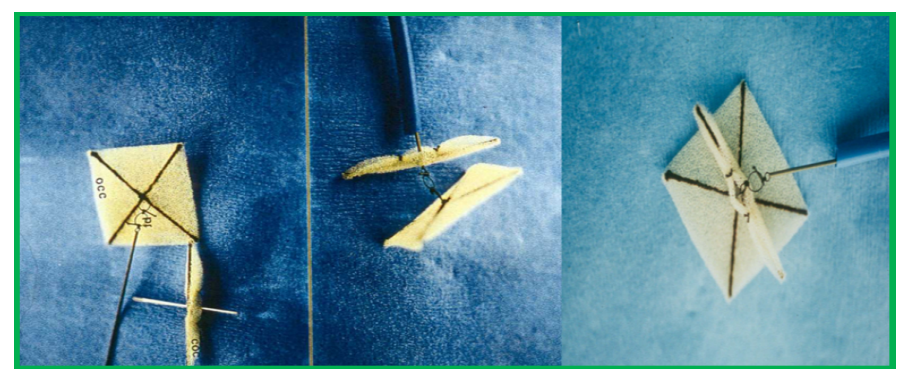

Figure 3. Photograph of a fourth-generation buttoned device



Figure 4. Photograph of a centering-on-demand buttoned device. A. centering mechanism is open; B. centering mechanism is closed

\section{EARLY INTERVENTION MAY BE BENEFICIAL}

Mayo Clinic - 123 Patients - Surgery 1956 -1960 27-Year Survival Rate

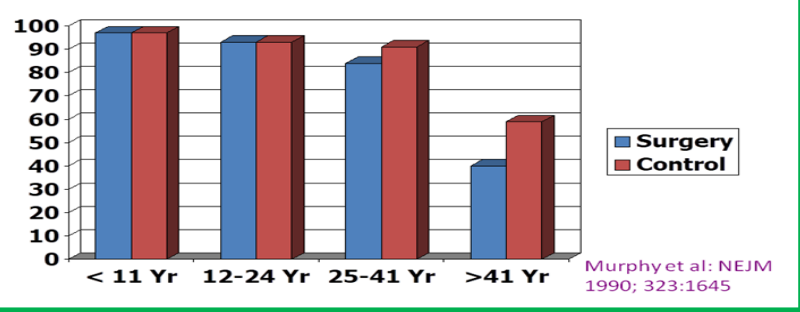

Figure 5. Bar diagram depicting 27-year survival rates following the closure of atrial septal defects by surgery; these data were compared with those of the normal population. If surgery was performed prior to 24 years of age, there was no significant difference in survival rates. However, the survival rates were lower when surgical closure was performed later. Constructed from the data of Murphy JG. et al., New Engl J Med, 1990; 323:1645-50. Reproduced from Rao PS, J Invasive Cardiol 2009; 21:76-82.

treatment for atrial defects [23-25]. The usual treatment of choice of moderate and large defects until recently is surgical correction. While surgical closure of ASDs is safe and effective with negligible mortality $[26,27]$, the morbidity associated with sternotomy/thoracotomy cannot be avoided. As a result, extensive efforts have been made by cardiologists to develop a non-surgical, catheter-based methods for ASD closure. Since the first description in the mid-1970s by King \& Mills and their associates [28-30] of an ASD occluding device, a number of other devices, as reviewed elsewhere [31-33] emerged. Despite the description of many different devices, the Amplatzer Septal Occluder (ASO), Amplatzer Cribriform Device, Gore HELEX ${ }^{\bullet}$ device, GORE ${ }^{\star}$ CARDIOFORM ASD Occluder are the only devices that are approved for general clinical use by the US Food and Drug Administration (FDA) in the USA. Occlutech Septal Occluder with design features similar to the ASO is used outside the USA. Accordingly, two methods, namely, surgical and transcatheter closure, are now available for ASD occlusion.

Surgical vs. Transcatheter Closure: While there are a limited number of studies comparing surgical with device closure, the existing studies suggest similar effectiveness [34,35]. Yet, the device closure is less invasive and requires no cardio-pulmonary bypass. In addition, the device occlusion appears to require shorter hospital stay (1 day vs. 4.3 days), has fewer number of complications ( $10 \%$ vs. $31 \%)$, and is less expensive (US \$11,000 vs. \$21,000) [36]. Additional disadvantages of surgery are the residual scar and psychological trauma to the patient and/or their parents. The overall assessment is that the device closure methods were demonstrated to be safe, cost-effective and favorably compare with surgical closure [34-37]. Transcatheter device occlusion of ASDs is now an established practice in most cardiac centers providing state of the art care to patients with CHD [37-41]. At the present time, ASD repair by surgery is mostly reserved for subjects 
whose ASDs have deficient septal rims in whom the defect is difficult to close with transcatheter methods or has had unsuccessful attempts to transcatheter occlude the defect. Furthermore, if intra-cardiac repair of other CHDs is contemplated, surgical closure of ASD can be performed at the same time.

\section{Transcatheter closure of ASD or patent foramen ovale for the prevention of recurrence of paradoxical embolism}

Transcatheter closure of the patent foramen ovale (PFO) with the buttoned device for the prevention of the recurrence of paradoxical embolism in adults was first performed by the author in 1992 [42,43], with subsequent reports on larger numbers of patients $[1,44,45]$. Some studies, as referenced in our paper [45], have suggested that paradoxical embolism through a PFO may be an important cause of cerebrovascular accidents (CVAs)/transient ischemic attacks (TIAs) in young adults. While there was no consensus, some physicians recommended surgical closure of PFOs/ASDs to prevent recurrence of paradoxical embolism and CVAs/TIAs as a substitute to life-long anticoagulation. Nonsurgical transcatheter occlusion of such defects was proposed as an alternative option to surgery by several cardiologists, including our group $[1,45]$. The author has previously described the experience with the closure of such defects in the international study cohort [1] and a detailed analysis of such patients at a single institution [45] which will be reviewed here.

Historical aspects: The first report of percutaneous closure of an atrial defect in a patient with CVA was with King's device in 1976 $[29,30]$. Many years later, Bridges [46], Rao [42,43] and their associates concurrently reported the use of the clamshell and buttoned devices, respectively, to close PFOs presumed to be the seat of paradoxical embolism. This was followed by use of this concept and method of PFO occlusion by a number of investigators, as referenced elsewhere $[45,47]$. The majority of the devices used for occluding ASDs [31-33] were used to close PFOs as and when the devices became available. Some of the ASD-occluding devices were modified and some new devices were designed specifically to address the anatomic features of the PFO; these include the Amplatzer PFO occluder, Cardia devices (PFO-Star and several of its subsequent generations), Premere occluder, Coherex Flat stent, PFx Closure System (which is not a device, but employs monopolar radio frequency energy to effect the closure of a PFO by welding the tissues of the septum primum with the septum secundum), pfm PFO-R, Solysafe PFO occluder, and others. The reader is referred to a review of this material published elsewhere $[32,33,48]$.

University of Wisconsin study: Patient Selection. Patients who had CVAs were thoroughly investigated by their respective neurologists, and no other cause was detected. Transesophageal echo (TEE) contrast studies with Valsalva were performed, and these revealed right-to-left shunting across the atrial septum (Figure $6 \mathrm{a}, \mathrm{b} \& \mathrm{c}$ ). The closure of the atrial defects was recommended by the patients' respective primary neurologists. Surgical and transcatheter options were presented to the patients/parents, and they opted for the experimental transcatheter occlusion.

Study subjects: During a 4.2 year period preceding September 1994, 10 patients aged 16 to 58 years (mean of 40 years) with weights ranging from 60 to $128 \mathrm{~kg}$ (mean of $77 \mathrm{~kg}$ ) had their PFOs/ASDs closed with the buttoned device to prevent the recurrence of presumed paradoxical embolism. There were seven females and three males. The immediate and short-term results of five of these patients have been included in our prior reports [42-44].

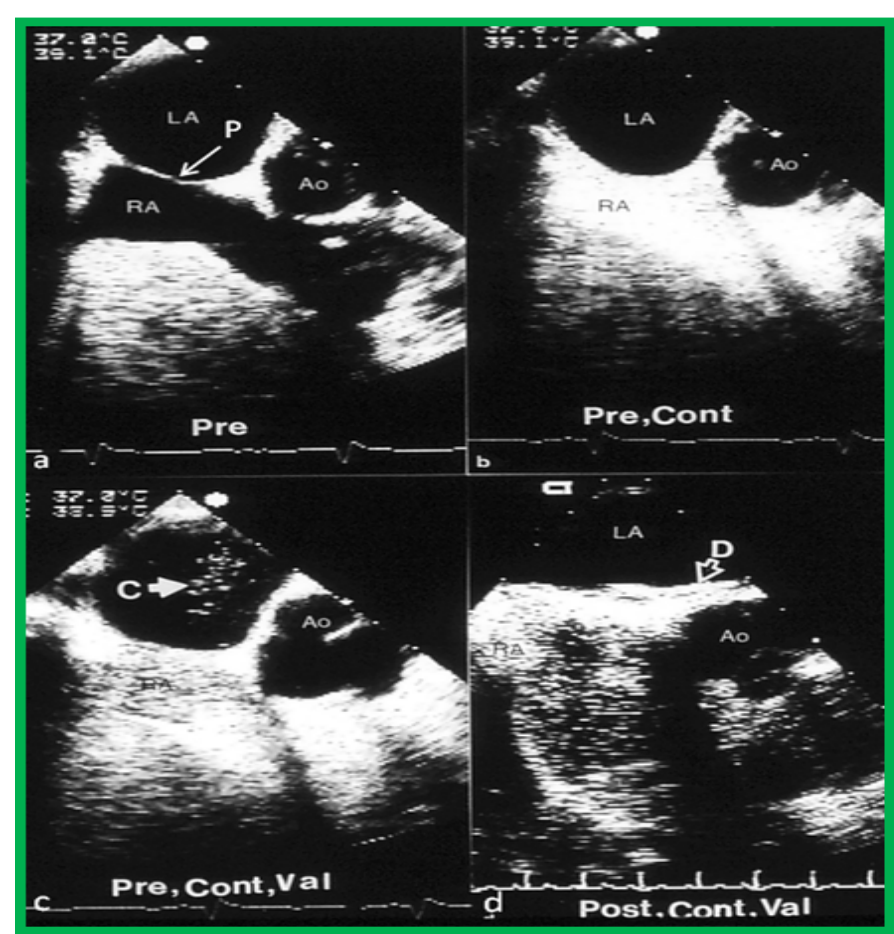

Figure 6. Selected video frames from a transesophageal echocardiographic study prior to (Pre) (a), and after contrast (cont) injection with agitated saline (b), after contrast with Valsalva (Val) (c), and following buttoned device closure of the patent foramen ovale (d). a. The patent foramen ovale (P) is shown by an arrow. b. The contrast study does not show bubbles in the left atrium (LA), c. The contrast study with Valsalva demonstrates contrast bubbles (C) in the LA, indicating a right-to-left shunt across the P. d. Following (Post) device (D) closure, no contrast bubbles were seen in the LA upon a contrast study with Valsalva. Ao, aorta; RA, right atrium. Rao PS, Ende DJ, Wilson AD, et al., Canad J Cardiol $1995 ; 11: 695-701$.

Protocol: The procedure was performed under a protocol for the use of custom-made devices in human subjects, approved by our IRB in the first patient. The remaining nine buttoned device occlusion procedures were performed under an FDA-supervised clinical trial with investigational Device Exemption (IDE) and were approved by the local Institutional Review Board (IRB). Informed consent was secured from the parents or from the patients, as appropriate. The method of device implantation and other procedural details were similar to those used with the other buttoned device occlusions, including the measurement of the stretched ASD diameter [1,5,7].

ASDs/PFOs: Six patients were found to have a PFO; two with an associated atrial septal aneurysm. Four were found to have a small fenestrated secundum ASD with a bidirectional shunt (minimal left-toright shunt but with predominant right-to-left shunt).

Devices: The buttoned devices that were implanted were between 30 and $40 \mathrm{~mm}$ in size; $35 \mathrm{~mm}$ was the most common size used $(\mathrm{n}=7)$.

Immediate results: Nine (90\%) of the 10 atrial defects were successfully closed with the buttoned devices. In each of these patients, the position of the device was stable (Figure 7), without a residual shunt, documented by TEE with contrast (Figure 8). In the remaining patient $(10 \%)$, because of the repeated slippage of the counter-occluder into the left atrium, the device was retrieved by transcatheter technique and surgical closure of the defect was performed successfully.

Follow-up results: The follow-up duration varied from 12 to 60 months (mean, $32 \mathrm{mo}$ ). None of the patients experienced the recurrence of CVAs/TIAs. On the basis of the results of transthoracic/ 




Figure 7. Transesophageal echocardiographic study demonstrating a good position of the buttoned device. The arrow points to the occluder $(\mathrm{O})$ along the left atrial (LA) side of interatrial septum. Reproduced from Ende DJ, Chopra PS, Rao PS, Am J Cardiol 1996; 78:233-6.

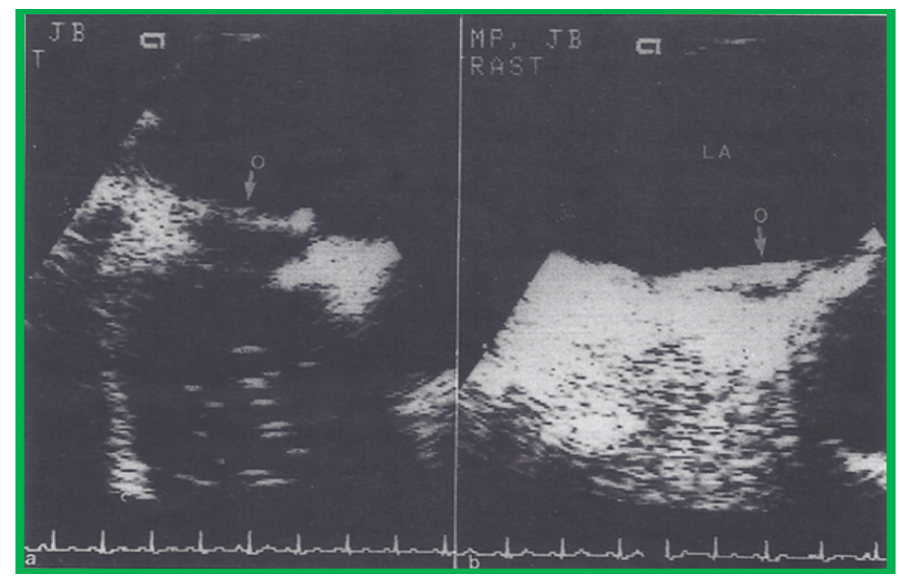

Figure 8. Successful closure of a patent foramen ovale, as demonstrated by contras transesophageal echocardiography. a. The occluder $(\mathrm{O})$ of the device in a good position along the left atrial (LA) side of the interatrial septum with contrast bubbles beginning to appear in the right atrium. b. Opacification of the right atrium by contrast material, with no crossing into the left atrium at the level of the fossa ovalis (the arrow points to the left atrial side of the occluder $(\mathrm{O})$. The latter was performed using the Valsalva manoeuvre during the contrast study. Reproduced from Ende DJ, Chopra PS, Rao PS, Am J Cardiol 1996; 78:233-6.

transesophageal echo contrast follow-up studies with Valsalva (Figure 6d), it was shown that right-to-left atrial shunts disappeared after one to six months in seven $(78 \%)$ of the nine patients. In two $(22 \%)$ patients, the shunt had disappeared at a TEE study 11 and 12 months after device implantation, respectively. In summary, during a follow-up period of up to 60 months, there was no recurrence of CVAs/TIAs, and the right-to-left shunt had disappeared in all patients, as measured by a sensitive method. One patient had an echo-dense structure on the left atrial side of the device on follow-up TEE, performed 23 months after a successful and uneventful PFO closure. This study revealed a $1.2 \times 0.8$ $\mathrm{cm}$ smooth mass along the lower border of the left atrial aspect of the device (Figure $9 \mathrm{a}$ and $\mathrm{b}$ ). There was no residual shunt at that time; the shunt was documented by contrast trans-thoracic echocardiography to be fully closed after one month following the device closure. The patient had no symptoms, but Warfarin therapy was restarted. A repeat TEE four months later demonstrated a smaller denser mass (Figure 9c), and the Warfarin was stopped. No clinical problems occurred during 10 months of follow-up after discontinuing anticoagulation. This was interpreted to be an instance in which a thrombus was caught passing through a PFO and became entrapped by the device and was subsequently endothelialized. [45]

In the discussion following the presentation of the data [45], we commented that a firm diagnosis of paradoxical embolism is frequently problematic, and the diagnosis is presumptive, making therapeutic decisions difficult. Recommendations varied from aspirin or Warfarin to transcatheter or surgical closure. There were no data regarding the long-term efficacy of these approaches in the prevention of recurrent CVAs/TIAs. There is indeed documentation of stroke recurrence in patients with patent foramina ovalia on chronic anticoagulation. In the study of transcatheter closure of PFOs with the clamshell device, there were no strokes at a mean follow-up of 8.4 months [46]. We further stated that transcatheter closure of PFOs/ASDs represents a recent approach to the treatment of this problem - a potential solution which would avoid the morbidity and mortality of either the medical or surgical approaches, and may offer better protection against recurrent events than does chronic anticoagulation.

International study: In the international study [1], there were 12 patients whose PFOs/ASDs were closed with the buttoned device for the prevention of the recurrence of paradoxical embolism. The device implantation was successful in all 12 patients. During follow-up, no recurrence of CVAs/TIAs was observed in 11 of 12 patients. TEE with contrast material and Valsalva, performed in seven patients six months after device implantation, revealed no residual shunt. One patient experienced a TIA, and TEE with contrast and Valsalva revealed a small residual right-to-left shunt. The patient had surgical closure of the PFO at the discretion of the primary cardiologist. No episodes of endocarditis or thrombus formation were seen [1].

Conclusion: On the basis of the data presented, we concluded that transcatheter occlusion of PFOs/ASDs is feasible, safe, and effective in preventing the recurrence of paradoxical embolism and CVAs. We suggested that anticoagulation should be continued until the disappearance of the right-to-left shunt across the atrial septum is demonstrated by contrast echocardiography using the Valsalva manoeuvre. Clinical trials on larger groups of patients are necessary to confirm the safety and efficacy of this method of management.

Additional comments: Subsequent to the above studies, a number of randomized clinical studies comparing medical treatment with transcatheter closure of atrial defects have been undertaken. These include CLOSURE I (STARFlex device), REDUCE (HELEX Septal Occluder), RESPECT (Amplatzer PFO Occluder device), and other

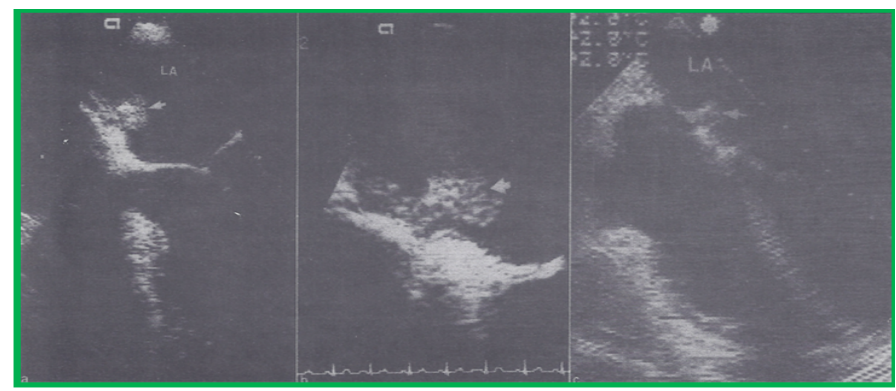

Figure 9. A. Selected video frame from a transesophageal echocardiographic study during routine follow-up at 23 months after the buttoned device closure procedure, demonstrating a smooth echo-dense structure along the occluder on the left atrial side of the interatrial septum (arrow), b. a close-up view of the same mass, likely representing an organized thrombus, c. a smaller and denser mass on transesophageal study after the patient had received Warfarin for four months. LA, left atrium. Reproduced from Ende DJ, Chopra PS, Rao PS, Am J Cardiol 1996; 78:233-6. 
trials. A review of the results of the RESPECT trial suggests that the closure of a PFO using the Amplatzer PFO Occluder is better than medical management in decreasing the risk of recurrent strokes in subjects with presumed paradoxical embolism [49]. Presumably based on these results, the US FDA approved the Amplatzer PFO Occluder in 2017 for clinical use for this purpose. Following the discontinuation of the buttoned device as mentioned above, the author has used the Amplatzer Cribriform Device for the closure of PFOs presumed to be causing paradoxical embolism. Now, the Amplatzer PFO Occluder, now that it is approved by the FDA may be used for this purpose.

\section{ASDs/PFOs with right-to-left shunt}

Although the buttoned device is effective in occluding secundum ASDs with a left-to-right shunt [1-5] and PFOs presumably responsible for paradoxical embolism $[44,45]$, it appears to have limitations in effectively occluding ASDs with an obvious right-to-left shunt [44]. In the first two situations, the pressure in the left atrium is higher than that in the right atrium and consequently, it is anticipated that the occlude will be held opposed to the atrial septum, pending the endothelialization of the device and its incorporation into the atrial septum. In right-toleft shunt lesions, since the right atrial pressure is higher than that in the left atrium, the occluder on the left atrial side may not be held in place, opposed to the atrial septum. This may potentially result in a residual right-to-left shunt. The buttoned device was modified to circumvent this problem; the square-shaped occluder component of the device is positioned on the right atrial side, and the single stand counteroccluder is designed to be on the left atrial side (Figure 10), hence its name - the inverted buttoned device [50]. The inverted buttoned device was studied to demonstrate its feasibility, safety, and effectiveness in occluding atrial defects with right-to-left shunts [50,51]. Adult patients who have had prior surgical repair of cyanotic CHDs, and who have residual atrial right-to-left shunts with significant hypoxemia, may also benefit from transcatheter occlusion of their ASDs/PFOs; the results of such studies have included both adult and pediatric patients [50,51]; therefore, the results will only be summarized.

Following inverted buttoned device implantation across the ASD/ $\mathrm{PFO}$, the systemic arterial oxygen saturation increased $(\mathrm{p}<0.001)$ from $82 \pm 7 \%$ (a range of $72-90 \%$ ) to $94 \pm 3 \%$ (a range of $88-98 \%$ ) immediately after occlusion (Figure 11$)$. The Qp:Qs $(0.62 \pm 0.15$ vs. $0.95 \pm 0.07 ; \mathrm{p}<0.001)$ and systemic venous saturation $(62 \pm 7 \%$ vs. $71 \pm$ $5 \% ; \mathrm{p}<0.01)$ increased, while the arterial-venous difference in oxygen content ( $45 \pm 18$ vs. $48 \pm 17 \mathrm{ml} / \mathrm{L}$ ) and systemic oxygen transport (573 \pm 119 vs. $594 \pm 140 \mathrm{ml} / \mathrm{min} / \mathrm{m} 2)$ did not significantly change $(\mathrm{p}>0.1)$ following ASD/PFO occlusion. At 6 to 18 month follow-up (median 12 months) a decrease in cyanosis and improved exercise tolerance were seen. The systemic arterial oxygen saturations $(92 \pm 3 \%)$ measured by pulse oximetry remained improved (Figure 12).

Based on these observations, it was concluded that transcatheter inverted buttoned device occlusion of ASDs/PFOs with right-to-left shunts is feasible in alleviating arterial hypoxemia and in reducing the risk of paradoxical embolism. It was suggested that further clinical trials on a larger number of patients, and the careful evaluation of follow-up results to examine the safety and long-term effectiveness of this procedure, are needed [50,51].

\section{Platypnea-orthodeoxia syndrome}

Platypnea-orthodeoxia syndrome (POS) is a rare condition seen in elderly subjects with incapacitating symptoms of dyspnea and arterial desaturation in an upright position [52,53]. Studies on transcatheter



Figure 10. Photograph of an inverted button device. BL. button loop; OCC, occluder; COc, counter occluder. Reproduced from Reference 33.



Figure 11. Results of inverted button device occlusion of right-to-left atrial shunts. Systemic arterial oxygen saturations before (Pre) (red circles) and immediately after (Post) (blue circles) device implantation across the atrial septum are shown, demonstrating an increase in the O2 saturation ( $\mathrm{p}<0.001)$. Modified from Rao PS, et al., Am J Cardiol 1997; 80:914-21.



Figure 12. Results of inverted button device occlusion of right-to-left atrial shunts. The systemic arterial oxygen saturations before (Pre) (open circles) and immediately after (Post) (closed circles) device implantation across the atrial septum are shown, demonstrating an increase in the saturation $(\mathrm{p}<0.001)$. During follow-up (FU) over 6 to 18 months (median 12 ) the saturations (triangles) remained high $(\mathrm{p}<0.01)$. Reproduced from Rao PS, et al., Am J Cardiol 1997; 80:914-21.

management of POS have been published by the author and his associates [52-54]. In some POS patients, the symptoms appear to be secondary to an increase in the right-to-left shunt across an ASD/PFO upon assuming an upright position. In the past, the closure of the ASD/ PFO by surgery was recommended to address this problem. We used the buttoned device to occlude ASDs/PFOs instead of surgical intervention $[52,53]$. The buttoned device was studied to demonstrate the feasibility, 
safety, and effectiveness of occluding ASDs/PFOs to relieve hypoxemia associated with POS [52].

Patient selection: Patients with clinical features of POS with a right-to-left shunt across their ASDs/PFOs demonstrable by TEE studies with contrast material were included in this study. Subjects with ostium secundum ASDs with left-to-right shunts, those with PFOs/ ASDs who had CVAs/TIAs, presumably secondary to paradoxical embolism, and those with PFOs/ASDs with right-to-left shunts in association with other congenital heart defects, were not included in this analysis.

Study subjects: Ten patients with POS underwent transcatheter buttoned device closure of their ASDs/PFOs during a 4-year period ending in January 2000. The age of the patients was $71 \pm 9$ years and varied between 60 and 83 years. There were six women and four men. Their weight was $55 \pm 11 \mathrm{~kg}$ with a range of 35 to $70 \mathrm{~kg}$. All the patients developed breathing difficulties upon sitting or standing (platypnea). Their arterial $\mathrm{O} 2$ saturation in the upright position was $76 \% \pm 7 \%$ and varied between $70 \%$ and $86 \%$ (orthodeoxia). Associated co-morbidities were present in all but one patient [52]. As mentioned in the "Patient Selection" section, all 10 patients had TEE with contrast material, which demonstrated a right-to-left shunt across the atrial septum. Cardiac catheterization data were normal in eight patients. In the remaining two patients, elevated pulmonary artery pressure was found: $85 / 40$ and $76 / 36 \mathrm{mmHg}$, respectively.

Protocol: Percutaneous closure of ASD/PFO was performed under a protocol approved by the FDA with an IDE. The protocol was also approved by the IRBs at Saint Louis University Health Sciences Center, St Louis, MO and Massachusetts General Hospital, Boston, MA. Informed consent was secured from each patient.

ASDs/PFOs: The diameter of the ASD/PFO, measured by TEE, was $8 \pm 3 \mathrm{~mm}$ with a range of 2 to $10 \mathrm{~mm}$. The balloon-stretched diameter was $12 \pm 3 \mathrm{~mm}$, with a range of 10 to $15 \mathrm{~mm}$.

Devices: Fourth-generation buttoned devices (Figures $2 \mathrm{C}$ and Figure 3) were implanted across the atrial septum in two patients. The square-shaped occluder was positioned on the left atrial side while the rhomboid-shaped, single-strand counter-occluder was placed on the right atrial side, and the two were buttoned together. Hybrid devices (Figure 13) [8] were implanted in the remaining eight patients (Figure 14). In the hybrid device, both the occluder and counter-occluder are square-shaped and there were two radiopaque buttons, as in the fourth-generation buttoned device, to ensure adequate buttoning.

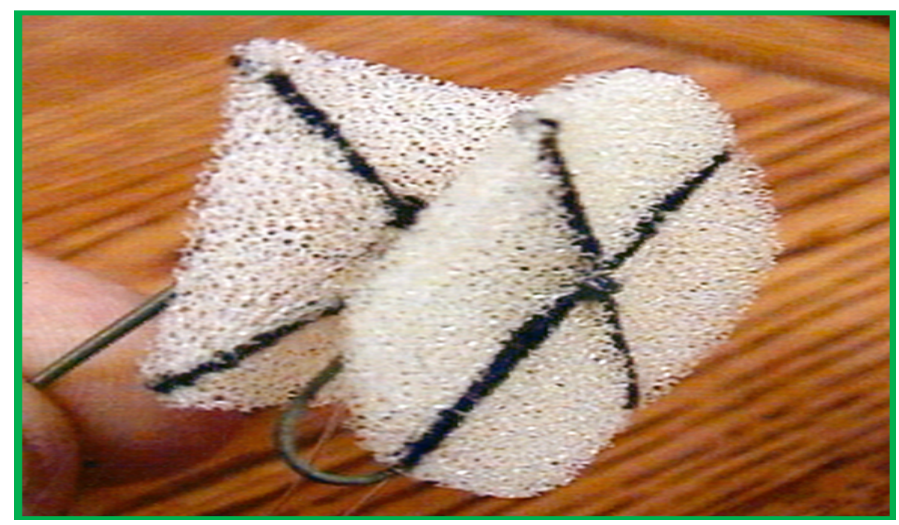

Figure 13. Photograph of a hybrid buttoned device. Note that both the occluder and counter occluder are square-shaped. Reproduced from Reference 33.

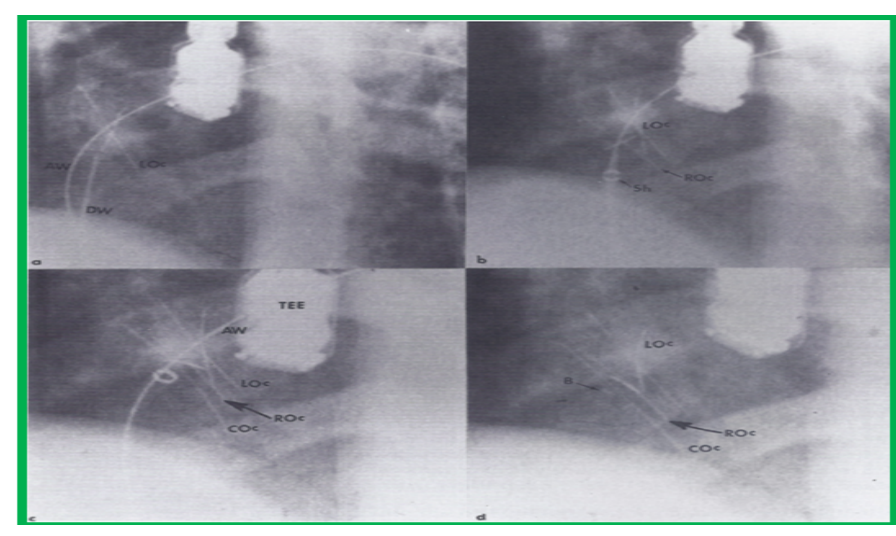

Figure 14. Selected cineradiographic frames from a left axial oblique view $\left(30^{\circ}\right.$ crania and $30^{\circ}$ left anterior oblique) obtained during various phases of hybrid buttoned device implantation. a. The left side occluder (LOc) is delivered into the left atrium by an overthe-wire technique. The Amplatzer guide wire (AW) used in the over-the-wire technique and the delivery wire (DW) are marked. $b$. This is followed by the delivery of the right atrial occluder (ROc). c. A counter-occluder (COc) is delivered on the right atrial side. d. The hybrid device after the removal of the DW and AW. Only one radiopaque button (B) is seen in this view. Sh, sheath; TEE, transesophageal echo probe. Reproduced from Rao PS, et al., Cathet Cardiovasc Intervent 2001; 54:77-82.

Immediate results: Successful device implantation was accomplished in all 10 patients. Contrast echocardiography demonstrated an improvement of the right-to-left shunt in all patients: a complete disappearance of the shunt was observed in five patients, while a trivial residual contrast bubble crossover was seen in the remaining five patients. The oxygen saturation in the upright position improved from $76 \pm 7 \%$ to $95 \% \pm 2 \%(\mathrm{p}<0.01)$ (Figure 15$)$. Clinically improved platypnea was seen, beginning the same afternoon or the morning following device implantation. No complications were encountered.

Follow-up results: The follow-up protocol was similar to that used in the other buttoned device implantations described in the preceding sections. The evaluation was focused on the relief of the symptoms of platypnea and on the improvement in oxygen desaturation in the upright position. Echocardiographic studies were performed to visualize the position of the device and to detect residual shunts by contrast. Follow-up data were available from 1 to 36 months, with a median of 12 months, following device implantation.

Symptomatic improvement continued in all but one patient. Repeat transthoracic $(\mathrm{n}=10)$ (Figure 16) and transesophageal $(\mathrm{n}=3)$ echo studies with contrast material (agitated saline) revealed residual shunts in two patients, but with acceptable oxygen saturation $(\geq 92 \%)$, measured by pulse oximetry, upon standing. The eight patients who had the hybrid buttoned device occlusion and one patient who had the fourth-generation buttoned device occlusion demonstrated no residual shunts, with acceptable $\mathrm{O} 2$ saturations, as mentioned above. One patient, who had fourth-generation buttoned device (without a square-shaped counter-occluder on the right atrial side) occlusion, demonstrated a significant residual right-to-left shunt with an arterial O2 saturation of $84 \%$ at a 1 -month follow-up examination. Because of these continued symptoms, a second buttoned device, this time an inverted device (Figure 10) with a $25 \mathrm{~mm}$ square-shaped counteroccluder on the right atrial side, was inserted across the residual defect, which resulted in improved symptoms and O2 saturation of $96 \%$. One month following the second device implantation, the patients showed persistent relief of both platypnea and orthodeoxia. 


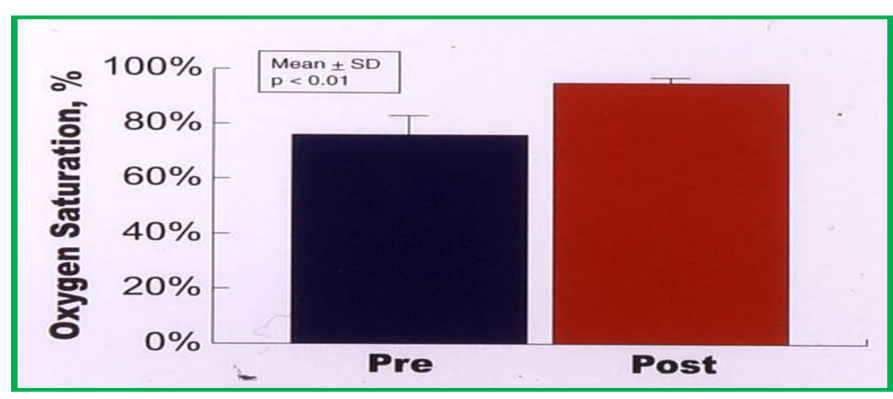

Figure 15. Bar graph demonstrating the increase $(p<0.01)$ in oxygen saturations in an upright position following buttoned device occlusion of atrial defects. Modified from Rao PS, et al., Cathet Cardiovasc Intervent 2001; 54:77-82.

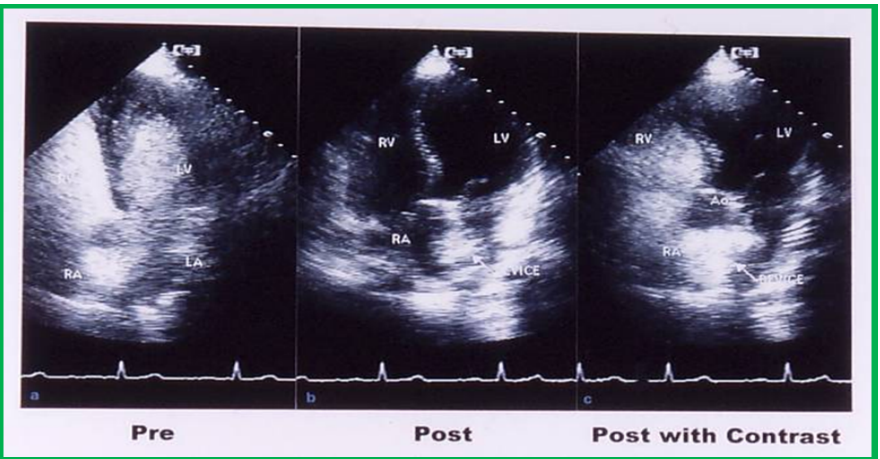

Figure 16. Selected video frame from apical views of a transthoracic echocardiographic study prior to (a) and during follow-up (post) (b and c). a. Prior to device closure, a contrast bubble study with agitated saline injection into a peripheral vein showed rapid opacification of the left atrium (LA) and left ventricle (LV). b. The DEVICE is seen across the atria septum. c. A repeat contrast bubble study with Valsalva, similar to that performed in (a) did not opacify the LA and LV, while densely opacifying the right atrium (RA) and right ventricle (RV), suggesting that there was no residual shunt across the atrial septum after the buttoned device occlusion of the atrial defect. Ao, aorta.



Figure 17. Selected video frames from a trans-esophageal echocardiogram following the implantation of an Amplatzer Septal Occluder to occlude an atrial septal defect, demonstrating the position of both disks in four chamber (A), bi-caval (B) and long axis (C) views. Note that the rims of the defect (thin arrows) are sandwiched between the left atrial (LA) and right atrial (RA) disks. LV, left ventricle; RV, right ventricle; SVC, superior vena cava. Reproduced from Reference 39.

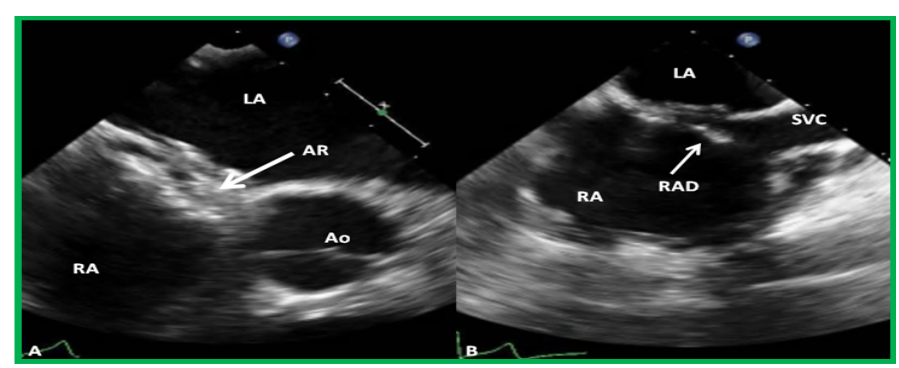

Figure 18. Selected two-dimensional video frames from trans-esophageal echocardiographic study in short axis (A) and bicaval (B) views demonstrating the position of the GORE CARDIOFORM ASD Occluder device following its implantation. The aortic rim (AR) (arrow in A) is seen sandwiched between the right and left atrial discs (A). In the bicaval view (B) there was some splaying of the superior portion of the right atrial disc (RAD). Ao, aorta; LA, left atrium; RA, right atrium; SVC, superior vena cava.

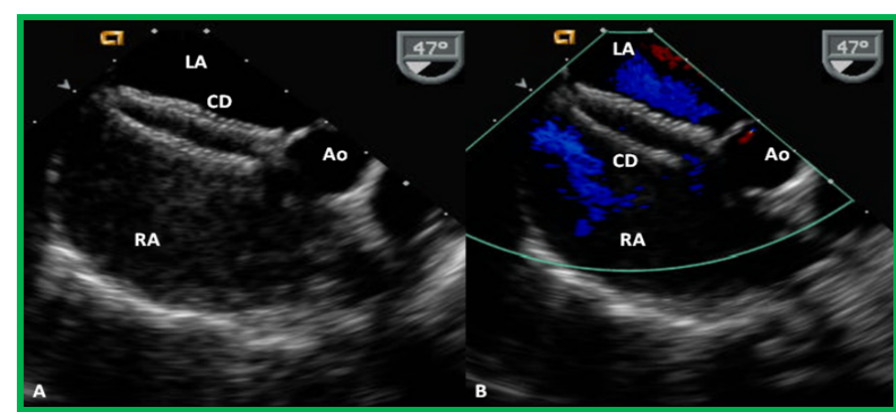

Figure 19. Selected video frames from a trans-esophageal echocardiogram following the delivery of an Amplatzer cribriform device (CD) demonstrating the position of both disks across the atrial septum (A) without any residual shunt (B) in a patient with a fenestrated atrial septal defect, illustrated in Figures 14-67 and 14-68. Ao, aorta; LA, left atrium; RA, right atrium. Reproduced from Reference 39.



Figure 20. Selected frames from cineflurograms in left axial oblique $\left(30^{\circ}\right.$ left anterior oblique and $30^{\circ}$ cranial) (A) and straight lateral (B) projections, showing the position of the Amplatzer cribriform device (CD) across the fenestrated atrial septal defect in the patient shown in Figures 14-67, 14-68 and 14-69. Note the thin waist (arrows in A and B) connecting the left and right atrial disks. While the mechanism for atrial septal occlusion using the Amplatzer Septal Occluder is the stenting of the atrial septal defect with the waist of the device, the mechanism for the occlusion of a fenestrated atrial septal defect is the covering of the defect with the disks of the CD. TEEP, trans-esophageal echocardiography probe. Reproduced from Reference 39.

Conclusions: On the basis of these data, it was concluded that buttoned device occlusion of ASDs/PFOs to relieve hypoxemia of POS is feasible, safe, and effective, and is an excellent alternative to surgical closure. It would appear that that a right atrial occluder such as those used in hybrid and inverted buttoned devices may be necessary to effectively occlude right-to-left shunts [52].

\section{Post-buttoned device approach}

Subsequent to the withdrawal of the buttoned device from clinical trials, as reviewed in Part I of these reviews, the author has used the Amplatzer Septal Occluder (Figure17) and GORE ${ }^{\bullet}$ CARDIOFORM ASD Occluder (Figure 18) to occlude ostium secundum ASDs and Amplatzer Cribriform Device (Figures 19 and 20) and Amplatzer PFO occluder to close PFOs producing 1. right-to-left shunts in patients who were previously treated for complex congenital cardiac anomalies, 2. forming the seat of paradoxical embolism resulting in CVAs/TIAs, and 3. causing right-to-left shunts in an upright position in platypneaorthodeoxia syndrome of the elderly.

\section{Conflict of interest}

The author has no potential conflict of interest in connection with this article.

\section{References}

1. Rao PS, Sideris EB, Hausdorf G, Rey C, Lloyd TR, et al. (1994) International experience with secundum atrial septal defect occlusion by the buttoned device. $\mathrm{Am}$ Heart J 128: 1022-1035. [Crossref] 
2. Lloyd TR, Rao PS, Beekman RH III, Mendelsohn AM, Sideris EB (1994) Atrial septal defect occlusion with the buttoned device: A multi-institutional U.S. trial. Am J Cardiol 73: 286-291. [Crossref]

3. Zamora R, Rao PS, Lloyd TR, Beekman RH III, Sideris EB (1998) Intermediate-term results of Phase I FDA Trials of buttoned device occlusion of secundum atrial septal defect. J Am Coll Cardiol 31: 674-676. [Crossref]

4. Sideris EB, Rao PS (1996) Transcatheter closure of atrial septal defects: Role of buttoned devices. J Invasive Cardiol 8: 289-296. [Crossref]

5. Rao PS, Berger F, Rey C, Haddad J, Meier B, et al. (2000) Results of transvenous occlusion of secundum atrial septal defects with $4^{\text {th }}$ generation buttoned device: Comparison with $1^{\text {st }}$, $2^{\text {nd }}$ and $3^{\text {rd }}$ generation devices. J Am Coll Cardiol 36: 583-592. [Crossref]

6. Rao PS, Sideris EB (2001) Centering-on-demand buttoned device: Its role in transcatheter occlusion of atrial septal defects. J Intervent Cardiol 14: 81-89. [Crossref]

7. Rao PS, Sideris EB (1998) Buttoned device closure of the atrial septal defect. $J$ Intervent Cardiol 11: 467-484.

8. Rao PS (2003) Buttoned device. In: Rao PS, Kern MJ. (editors) Catheter Based Devices for Treatment of Noncoronary Cardiovascular Disease in Adults and Children. Lippincott, Williams \& Wilkins, Philadelphia, PA, 17-34.

9. Rao PS, Sideris EB (2003) Buttoned device modifications: Influence on feasibility, safety and effectiveness. Poster presentation at the 26th Annual Scientific Sessions of the Society of Cardiac Angiography and Interventions. Cath Cardiovasc Intervent Westin Copley Place, Boston, MA, May 7-10, 59:153.

10. Rao PS (2020) Atrial septal defects. In: Rao PS. Pediatric Cardiology: How It Has Evolved Over the Last 50 Years. Cambridge Scholars Publishing, New Castle upon Tyne, 321-386.

11. Ward C (1994) Secundum atrial septal defect: Routine surgical treatment is not of proven benefit. Br Heart J 71: 219-223. [Crossref]

12. Gatzoulis MA, Redington AN, Somerville J, Shore DF (1996) Should atrial septal defects in adults be closed? Ann Thorac Surg 61: 657-659. [Crossref]

13. Webb G (2001) Do patients over 40 years of age benefit from closure of an atrial septal defect? Heart 85: 249-250. [Crossref]

14. Rao PS (2009) When and how should atrial septal defects be closed in adults. J Invasive Cardiol 21: 76-82. [Crossref]

15. Rao PS (2012) Why, When and how should atrial septal defects be closed in adults. In: Rao PS, editor, Atrial Septal Defect, ISBN 978-953-51-0531-2; InTech, Rijeka, Croatia, pp: $121-138$

16. Rosas M, Attie F, Sandoval J, Castellano C, Buendía A, et al. (2004) Atrial septal defect in adults $\geq 40$ years old: Negative impact of low arterial oxygen saturation. Inter J Cardiol 93: 145-155.

17. Horvath KA, Burke RP, Collins JJ Jr, Cohn LH (1992) Surgical treatment of adult atrial septal defect: Early and long-term results. J Am Coll Cardiol 20: 1156-1159. [Crossref]

18. Konstantinides S, Geibel A, Olschewski M, Görnandt L, Roskamm H, et al. (1995) A comparison of surgical and medical therapy for atrial septal defects in adults. $N$ Engl $J$ Med 333: 469-473. [Crossref]

19. Eidem BW, O'Leary PW, Tei C, Seward JB (2000) Usefulness of the myocardial performance index for assessing right ventricular function in congenital heart disease. Am J Cardiol 86: 654-658. [Crossref]

20. Salehian O, Horlik E, Schwermann M, Haberer K, McLaughlin P, et al. (2005) Improvement in cardiac form and function after transcatheter closure of secundum atrial septal defects. J Am Coll Cardiol 45: 499-504. [Crossref]

21. Brochu MC, Baril JF, Dore A, Juneau M, De Guise P, et al. (2002) Improvement in exercise capacity in asymptomatic and mildly symptomatic adults after atrial septal defect percutaneous closure. Circulation 106: 1821-1826. [Crossref]

22. Murphy JG, Gersh BJ, McGoon MD, Mair DD, Porter CJ, et al. (1990) Long-term outcome after surgical repair of isolated atrial septal defect. New Engl J Med 323: 1645-1650. [Crossref]

23. Kirklin JW, Swan HJ, Wood EH, Burchell HB, Edwards JH (1955) Anatomic, physiologic, and surgical considerations in repair of interatrial communications in man. J Thorac Surg 29: 49-53. [Crossref]

24. Rao PS (1981) Present status of surgery in congenital heart disease. Indian J Pediatr 49: 349-463. [Crossref]

25. Chopra PS, Rao PS (1991) Surgical management of congenital heart disease: Current trends. Indian J Pediat 58: 623-640. [Crossref]
26. Galal MO, Wobst A, Halees Z, Hatle L, Schmaltz AA, et al. (1994) Perioperative complications following surgical closure of atrial septal defect type II in 232 patients a baseline study. Europ Heart J 15: 1381-1384. [Crossref]

27. Pastorek JS, Allen HD, Davis JT (1994) Current outcomes of surgical closure of secundum atrial septal defect. Am J Cardiol 74: 75-77. [Crossref]

28. King TD, Mills NL (1974) Nonoperative closure of atrial septal defects. Surgery 75: 383-388. [Crossref]

29. King TD, Thompson SL, Steiner C, Mills NL (1976) Secundum atrial septal defect: nonoperative closure during cardiac catheterization. J Am Med Assoc 235: 2506-2509. [Crossref]

30. Mills NL, King TD (1976) Nonoperative closure of left-to-right shunts. J Thorac Cardiovasc Surg 72: 371-378. [Crossref]

31. Chopra PS, Rao PS (2000) History of the development of atrial septal occlusion devices. Current Intervent Cardiol Reports 2: 63-69. [Crossref]

32. Rao PS (2003) History of atrial septal occlusion devices, In: Rao PS, Kern MJ, editors Catheter Based Devices in the Treatment of Non-coronary Cardiovascular Disease in Adults and Children, Lippincott, William \& Wilkins, Philadelphia, p.1-9.

33. Alapati S, Rao PS (2012) Historical aspects of trans-catheter occlusion of atrial septa defects. In: Rao PS, editor. Atrial Septal Defect, ISBN 978-953-51-0531-2; InTech, Rijeka, Croatia, p. 57-84.

34. Berger F, Vogel M, Alexi-Meskishvili V, Lange PE (1999) Comparison of results and complications of surgical and Amplatzer device closure of atrial septal defects. $J$ Thorac Cardiovasc Surg 118: 674-678. [Crossref]

35. Du ZD, Hijazi ZM, Kleinman CS, Silverman NH, Larntz K, et al. (2002) Comparison between transcatheter and surgical closure of secundum atrial septal defect in children and adults: Results of multicenter nonrandomized trial. J Am Coll Cardiol 39: 18361844. [Crossref]

36. Kim JJ, Hijazi ZM (2002) Clinical outcomes and costs of Amplatzer transcathete closure as compared with surgical closure of ostium secundum atrial septal defects. Med Science Monitor 12: CR787-791. [Crossref]

37. Bettencourt N, Salome N, Carneiro F, Gonçalves M, Ribeiro J, et al. (2003) Atrial septal closure in adults: Surgery versus Amplatzer - Comparison of results. Rev Port Cardiol 22: 1203-1211. [Crossref]

38. Rao PS (2003) Catheter closure of atrial septal defects. J Invasive Cardiol 15: 398-400

39. Rao PS. Non-surgical closure of atrial septal defects in children. In: Atrial and Ventricular Septal Defects: Molecular Determinants, Impact of Environmental Factors and Non-Surgical Interventions, Larkin, SA (Ed), ISBN: 978-1-62618-326-1. Nova Science Publishers, Inc.

40. Rao PS, Harris AD (2017) Recent advances in managing septal defects: Atrial septal defects. F1000Res 6: 2042-2050. [Crossref]

41. Rao PS (2019) Management of congenital heart disease: State of the art-Part IAcyanotic heart defects. Children (Basel) 6: 42. [Crossref]

42. Rao PS, Wilson AD, Levy JM, Chopra PS (1992) Role of "buttoned" double-disk device in the management of atrial septal defects. Am Heart $J$ 123: 191-200.

43. Rao PS, Wilson AD, Chopra PS (1992) Transcatheter closure of atrial septal defect by "buttoned" devices. Am J Cardiol 69: 1056-1061. [Crossref]

44. Rao PS, Ende DJ, Wilson AD, Smith PA, Chopra PS (1995) Follow-up results of transcatheter occlusion of atrial septal defect with buttoned device. Canad J Cardiol 11: 695-701. [Crossref]

45. Ende DJ, Chopra PS, Rao PS (1996) Transcatheter closure of atrial septal defect or patent foramen ovale with the buttoned device for prevention of recurrence of paradoxic embolism. Am J Cardiol 78: 233-236. [Crossref]

46. Bridges ND, Hellenbrand W, Latson L, Filiano J, Newburger JW, et al. (1992) Transcatheter closure of patent foramen ovale after presumed paradoxical embolism. Circulation 16: 83-84. [Crossref]

47. Windecker S, Meier B (2003) Percutaneous closure of patent foramen ovale in patients with presumed paradoxical embolism. In: Rao PS, Kern MJ (eds): Catheter Based Devices for the Treatment of Noncoronary Cardiovascular Disease in Adults and Children. Lippincott, Williams \& Wilkins, Philadelphia, PA, 111-118.

48. Rao PS. History of trans-catheter interventions in pediatric cardiology. In: Vijayalakshm IB, ed. Cardiac Catheterization and Imaging (From Pediatrics to Geriatrics), Jaypee Publications, New Delhi, India, 2015:3-20. Chapter 1. 
49. Saver JL, Carroll JD, Thaler DE, Smalling RW, MacDonald LA, et al. (2017) Longterm outcomes of patent foramen ovale closure or medical therapy after stroke. $N$ Engl J Med 377: 1022-1032. [Crossref]

50. Rao PS, Chandar JS, Sideris EB (1997) Role of inverted buttoned device in transcatheter occlusion of atrial septal defect or patent foramen ovale with right-to-lef shunting associated with previously operated complex congenital cardiac anomalies. Am J Cardiol 80: 914-921. [Crossref]

51. Rao PS (2003) Transcatheter closure of atrial septal defects with right-to-left shunt. In: Rao PS, Kern MJ. (editors) Catheter Based Devices for Treatment of Noncoronary Cardiovascular Disease in Adults and Children. Lippincott, Williams \& Wilkins, Philadelphia, PA:119-28. Chapter 14
52. Rao PS, Palacios IF, Bach RG, Bitar SR, Sideris EB (2001) Platypnea-orthodeoxia syndrome: Management by trans-catheter buttoned device implantation. Cathet Cardiovasc Intervent 54: 77-82. [Crossref]

53. Bitar S, Rao PS (2003) Platypnea-orthodeoxia syndrome: Transcatheter management In: Rao PS, Kern MJ. (editors) Catheter Based Devices for Treatment of Noncoronary Cardiovascular Disease in Adults and Children. Lippincott, Williams \& Wilkins, Philadelphia, PA,129-32. Chapter 15.

54. Rao PS (2004) Transcatheter management of platypnea-orthodeoxia syndrome. $J$ Invasive Cardiol 16: 583-584. [Crossref]

Copyright: $(2020$ P. Syamasundar Rao. This is an open-access article distributed under the terms of the Creative Commons Attribution License, which permits unrestricted use, distribution, and reproduction in any medium, provided the original author and source are credited. 